\title{
Probabilistic Representation of 3D Object Shape by In-Hand Exploration
}

\author{
Diego R. Faria, Ricardo Martins, Jorge Lobo and Jorge Dias
}

\begin{abstract}
This work presents a representation of 3D object shape using a probabilistic volumetric map derived from inhand exploration. The exploratory procedure is based on contour following through the fingertip movements on the object surface. We first consider the simple case of having single hand exploration of a static object. The cumulative pose data provides a 3D point cloud that is quantized to the probabilistic volumetric map. For each voxel we have a probability distribution for the occupancy percentage. This is then extended to in-hand exploration of non-static objects. Since the object is moving during the in-hand exploration, and we also consider the use of the other hand for re-grasping, object pose has to be tracked. By keeping track of object motion we can register data to the initial pose to build a consistent object representation. An object centered representation is implemented using the computed object center of mass to define its frame of reference. Results are presented for in-hand exploration of both static and non-static objects that show that valid models can be obtained. The 3D object probabilistic representation can be used in several applications related with grasp generation tasks.
\end{abstract}

\section{INTRODUCTION}

$\mathrm{T}$ HE ability of manipulating different objects dexterously is one of the most well succeeded human skills. This skill is studied and pursued by researches in robotic field to endow a robot with this ability. Despite the different approaches found in the literature to try to imitate the human dexterity and also the advanced robotic hands developed using new technologies, there are still differences in humans and robots in handling tasks. The ability of a human manipulation involves different elements such as hand, arm, eyes or head where the human has many degrees of freedom and can easily deal with the control of those parts differently of the robots which is found nowadays, they do not have the control and skills as human do. Humans use multiple sensory information to recognize objects. The best view for object representation for the haptic modality, however, is the side the fingers naturally explore the most [1]. The studies of human experiences in grasping and object exploration tasks can be applied in robotic field, i.e., to endow a robot with similar skills through a generalization of these human abilities. The contour following is a usual way of "exploratory procedures" that people use for determining the

This work is partially supported by the European project: HANDLE, FP7-231640. Diego R. Faria and Ricardo Martins are supported by Portuguese Foundation for Science and Technology (FCT). Diego R. Faria, Ricardo Martins, Jorge Lobo and Jorge Dias are with Institute of Systems and Robotics, DEEC, University of Coimbra, Polo II, 3030-290, Coimbra, Portugal (e-mails: \{diego; rmartins; jlobo; jorge\}@isr.uc.pt). geometry of an object [2]. Haptic object recognition is presented by [3] using a dexterous robot hand with a manipulator arm. Through the hand contact by enclosing the objects at predefined positions it was possible evaluate joint angles and force readings. The object shape recovery was performed using sparse contact points from the hand. In [4] the object shape contour is acquired by a human hand using a data glove where the human operator visually guides the contact sensor along the contours of the object by the index fingertip. An extended superquadrics function is used for primitive modeling which can represent a variety of cubical and spherical geometries. In [5] superquadrics functions were used for shape recovery from haptic exploration using fingertip tactile sensors. The authors have applied a hybrid minimization method using a genetic algorithm by considering the contact normal information to recover superquadrics primitives. In [6] a haptic exploration procedure is presented using two $3 \mathrm{DoF}$ fingers and a fixed palm. The authors present a sequence of phases in which some fingers are responsible for grasping. The work presented in [7] considers the detection of small surface features, such as ridges and bumps. The features are based on the ratio of object surface curvature to the radius of the fingertip to acquire the data.

The purpose of this work is to use the in-hand exploration information to compute a probabilistic volumetric map for object-centric representation. Fig. 1 represents an overview of the contribution of this work as well as the future applications that can be developed from the results of this probabilistic representation of the object shape. In this work we are dealing with in-hand exploration of static or nonstatic objects. The proposed probabilistic representation can be used in the future for multisensory fusion to combine information acquired from in-hand exploration and visual perception. As shown in Fig.1, after acquiring the 3D object model it is possible to extract its geometrical primitives. Humans usually make identification of objects, segmenting some object parts to choose the best place to grasp. According to recognition by components theory (RBC) [8], humans are able to recognize objects by separating them into geometric icons to find graspable parts on the object. From the probabilistic representation of the object model we can find the region with higher probability that means the most explored region by a subject. Decomposing the volumetric model of the object in primitives we can also estimate the graspable part by taking into consideration the most explored region. We can also verify the first region grasped (contact points), it indicates the chosen part as a strong 
candidate to be the handle part of the object due to the fact that humans usually choose to grasp an object by its designed part for grasping or the easier part to grasp it.

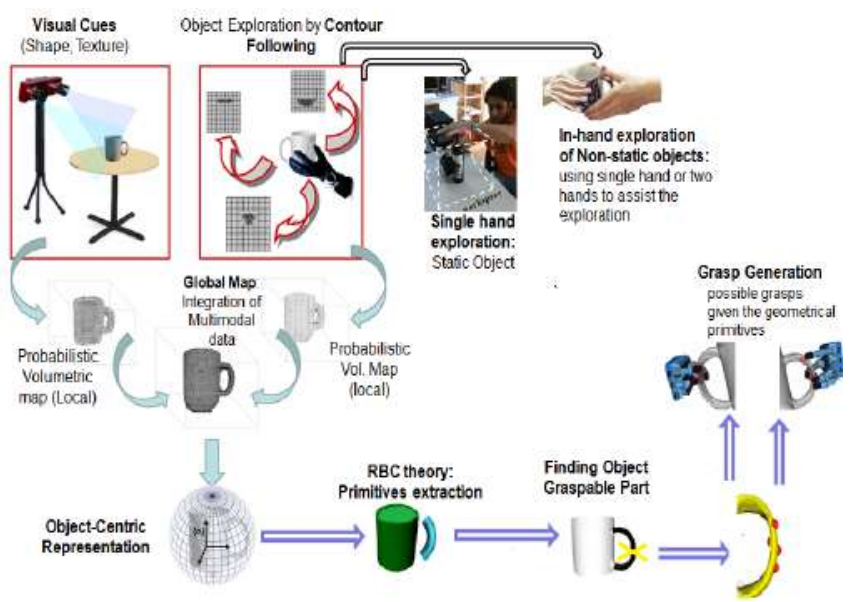

Fig. 1 - Overview of this work and link to future applications.

\section{SCENARIO AND CONTEXT}

For the exploratory procedure we are using the Polhemus Liberty electromagnetic motion tacking system [9]. The magnetic sensors were attached to the fingertips in order to perform the object contour following to acquire 3D point's cloud of the object surface. For this experiment, we decided to use the thumb, index and middle fingers to explore the object surface, since it should be enough to cover the object shape through the movements around it. In this scenario we are also using a stereo camera from Videre Design [10] to take a shot of the object shape before the in-hand exploration to keep the object texture. In this work we are not addressing sensor fusion to compute a probabilistic map using visual data, just extracting texture from vison system. Through a calibration of sensors (stereo camera and motion tracker) it is possible to have correspondence of the occupied voxels of the probabilistic map and the points of the extracted 3D data cloud points to show the probabilitic map in gray scale representing the probability of occupancy as well as the voxels with texture.

To reach the purpose of in-hand exploration, there are two possible contexts. The first one is the exploration of static object on a table delimiting the region (mapping area) where the object needs to be placed. In this first scenario, just one hand is used to explore the object (usually the right hand) and in most of the cases the left hand assists the task by fixing the object on its top part so that the object does not move during the exploration, see Fig. 1. The second way is to perform the in-hand exploration using a single hand or two hands (one of the hand hold the object while another one explores it). In this second case the object is allowed to move while the exploratory procedure is being performed. The object motion introduces new challenges to the task of computing the object volumetric map.

\section{A. Single Hand Exploration of Static Objects}

In order to acquire the probabilistic representation of an object using a volumetric map, it is necessary to know a priori an estimated area where the object is placed for mapping. Some problems are addressed in this task such as the object needs to be completely static. As long as the sensors we are adopting to extract information of the object pose is a magnetic tracker, some limitation needs to be taken into account. After the sensors initialization, 3D points are constantly acquired so that when the hands are performing the exploratory procedure, if the fingers movements are not on the object surface, those generated $3 \mathrm{D}$ points probably do not belong to the object shape. If the involuntary movements (out of the object surface) are sporadic, those 3D points will be ignored in the probabilistic map due to do not have higher probability in their occurrence (the volumetric representation is based on probabilities higher than a threshold: 0.7). However, if a finger movement occurs with higher frequency in the same region out of the object surface, it can harm the result. Other possibility of this happening is when someone is exploring an object and then it is moved from its original position. The final result will have a concentration of points representing the same part of the object in different positions. The solution is presented in the next subsection.

The setup for this experiment is composed of a wooden table, without any metallic parts, since the magnetic tracker is sensitive to nearby ferromagnetic materials. The rigid 3D object is fixed on the tabletop in a defined workspace. A workspace of $35 \mathrm{~cm}^{3}$ was defined on the table for the object mapping. Each voxel of the volumetric map was defined to represent an area $0.5 \mathrm{~cm}^{3}$ due to the sensor position resolution at $30 \mathrm{~cm}$ range is $\sim 1 \mathrm{~mm}$. During the exploratory procedure at short period of discrete intervals the volumetric map is updated with the sensors measurements. Fig. 2 shows the experimental setup area.

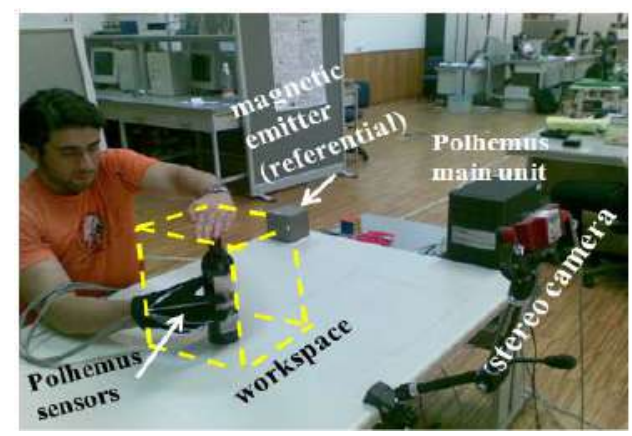

Fig.2. Experimental setup for single hand exploration.

\section{B. In-Hand Exploration of Non-static Objects}

The experimental setup for in-hand exploration of nonstatic objects follows the same structure of the single hand exploration. The difference about this new task is on the object position for mapping: the object does not need to be static anymore. Since the object is allowed to move, it is possible to use the other hand (usually left hand) to assist the right hand, holding the object for better exploration of the 
object. This task becomes more complex than the first one presented in the previous subsection due to the non-static objects. In this work the probabilistic map is used for the representation of the object shape so that there is a need to deal with the object rotations and translations during the inhand exploration. Knowing the object initial position and the object displacements, we can compute the transformations to have all point in the same frame of reference. Given that the sensor attached to the object has $6 \operatorname{DoF}\{\mathrm{x}, \mathrm{y}, \mathrm{z}$, yaw, pitch, roll $\}$, we can compute the rotations and translation of the object. Before computing the probabilistic map in this context, we compute the transformations to have all points of the object in the same frame of reference. We compute the rotation matrix of the object in a specific instant using $\alpha$ $=$ yaw (rotation in $z$ axis), $\beta=$ pitch (rotation in $y$ ) and $\varphi=$ roll (rotation in $x$ ).

To map the point cloud in the same frame of reference, for all points, we find the translation of the fingertip sensor to the object sensor and then we apply the rotation to that point:

$$
p^{\prime}=R_{o} t
$$

where $p^{\prime}$ is the new position of the 3D point that we are mapping to the same frame of reference of the object sensor; $R_{\mathrm{o}}$ is the rotation matrix $3 \times 3$ of the object sensor and $t$ the translation of the fingertip sensor to the object sensor.

\section{3D OBJeCt MOdEL REPRESENTATION}

Mapping techniques as occupancy grid [11], [12] has been used in robotics field to describe an environment of a mobile robot. Two-Dimensional grid has been used for static indoor mapping [13]. The idea is to verify the probability of each cell to be full or empty after the sensors observation. Probabilistic volumetric maps are also useful in robotics field as presented in [14] for data fusion (visual and auditory perception). The main motivations of using the probabilistic map are: simpler way of static object reconstruction and representation; the uncertainty of sensor noise due to real world (the sensor probability model depends on the characteristics of the sensor and the object being sensed).

\section{A. In-hand Exploration Sensor Model}

This subsection describes how to build the probabilistic map using fingertip pose data. For in-hand exploration a magnetic sensor is attached to the fingers to acquire the shape of an object by contour following. Each sensor returns the 3D coordinates based on sensors' frame of reference. The frame rate of each sensor was defined to be up to $15 \mathrm{~Hz}$. During the data acquisition, a workspace $(35 \mathrm{~cm} \times 35 \mathrm{~cm} \times$ $35 \mathrm{~cm}$ ) is defined in the experimental area for mapping. This space is subdivided in $0.5 \mathrm{~cm} \times 0.5 \mathrm{~cm} \times 0.5 \mathrm{~cm}$ voxels. During the displacement of each of the magnetic on the object surface, it is possible to identify in which cell that measurement is inserted. Due to the size of each cell relatively to the standard deviation of the magnetic tracking sensors measurements (up to $2 \mathrm{~mm}$ ), it is defined inside each cell a 3D isotropic Gaussian probability distribution, $P($ $\left.Z_{\text {grasp }} \mid O c\right)$, centred at the cell central point with standard deviation $0.2 \mathrm{~cm}$ and mean value equal to the cell central point coordinates of the cell. The probability that a measurement belongs to a cell is given by:

$P\left(Z_{\text {grasp }} \mid O_{C}\right)=\frac{1}{(2 \Pi)^{3 / 2}\left|\sum\right|^{1 / 2}} \exp \left(-\frac{1}{2}(\mathbf{x}-\boldsymbol{\mu})^{T} \Sigma^{-1}(\mathbf{x}-\boldsymbol{\mu})\right)$

where $P\left(Z_{\text {grasp }} \mid O_{c}\right)$ represents the probability distribution of the sensor measure given a specific cell $\mathrm{O}_{\mathrm{c}} ;\left|\sum\right|$ represents the determinant of $\sum$ (sensor noise variation). It can also represent a scalar value. Due to the normalization, (2) takes the form:

$$
P\left(Z_{\text {grasp }} \mid O_{C}\right)=\exp \left(-\left(\frac{\left(x-u_{x}\right)^{2}+\left(y-u_{y}\right)^{2}+\left(z-u_{z}\right)^{2}}{2 \sigma^{2}}\right)\right)
$$

\section{B. Probabilistic Volumetric Map Occupancy Estimation}

In our previous work [15], a probabilistic map was developed to represent objects shapes through grasp exploration, but with many limitations. We are supporting the current work on that idea of volumetric map, addressing the problems and limitations and extending this work to deal with those problems as described in section II. This work also presents how to employ the volumetric map for data fusion in the future, for more details see subsection C. The occupancy of each individual voxel is assumed to be independent from the other voxels' occupancy and thus $O_{C}$ is a set of independent random variables: $C \in M$ - index a cell on the Map; $O_{C}$ - probability value describing the occupancy of the cell $C$; $Z_{\text {grasp }}$ - grasp exploration measure that influences the cell $C . P\left(O_{C}\right)$ - Probability distribution of preliminary knowledge of coverage value describing the occupancy of the cell $C$, initially is an uniform distribution; $P\left(Z_{\text {grasp }} \mid O_{C}\right)$ - probability distribution corresponding to the set of measurement $Z_{\text {grasp }}$ that influences the cell $C$ taken from the in-hand exploration data. This distribution is taken from occupancy model. The probability distribution on the occupation's probability $P\left(O_{C} \mid Z_{\text {grasp }}\right)$ for each voxel is given by:

$$
\begin{aligned}
& P\left(O_{C} \mid Z_{\text {grasp }}\right)= \\
& \frac{P\left(Z_{\text {grasp }} \mid O_{C}\right) P\left(O_{C}\right)}{P\left(Z_{\text {grasp }} \mid O_{C}\right) P\left(O_{C}\right)+P\left(Z_{\text {grasp }} \mid \neg O_{C}\right) P\left(\neg O_{C}\right)}
\end{aligned}
$$

where $P\left(O_{C} \mid Z_{\text {grasp }}\right)$ is the posterior value; $P\left(Z_{\text {grasp }} \mid O_{C}\right)$ is acquired by the sensor measurement (likelihood computed through the sensor model); $P\left(O_{C}\right)$ is the prior information (at the beginning it is a uniform distribution representing the state full or empty) and subsequently the previous posterior for that cell becomes the prior for the next computation. 


\section{Probabilistic Map for Multimodal Data Fusion: Vision Model and Entropy as Confidence Level}

This subsection presents how to build the volumetric model for data fusion to be employed in future applications where will be used other sensor modality, e.g. vision to compute the occupancy probability in the map beside of the sensor for in-hand exploration. The variables presented in the subsection $B$ used in (4) to estimate the occupancy probability of each cell is kept. In case of using vision, the sensor model $P\left(Z_{\text {vision }} \mid O_{C}\right)$ needs to be defined. Visual systems usually are implemented as deterministic algorithms returning the visual properties like range values. In the 3D world we have the position as $\boldsymbol{X}_{\boldsymbol{k}}$ that represents the $\{x, y, z\}$ coordinates of a point $p$ and the range measurement $d_{j}$ taken by the sensor and the position magnitude of vectors $\vec{r}_{k, i}$ with the direction of the projection line corresponding to each measurement. Adopting the solution proposed in [16] we can have voxel's occupancy belief as Gaussian distribution as follows:

$$
P\left(O c \mid Z_{v i s}\right)=N\left(\mu_{c}\left(d_{j}, d_{j}^{l}\right), \sigma\left(d_{j}, d_{j}^{l}\right), O c\right)
$$

where

$$
\begin{gathered}
\mu\left(d_{j}, d_{j}^{l}\right)= \begin{cases}0, & \left(d_{j}^{l}-d_{j}\right) \leq-\frac{\varepsilon}{2} \\
\frac{1}{2}+\frac{d_{j}^{l}-d}{\varepsilon}, & \left|d_{j}^{l}-d_{j}\right|<\frac{\varepsilon}{2} \\
1, & \left(d_{j}^{l}-d_{j}\right)>\frac{\varepsilon}{2}\end{cases} \\
\sigma\left(d_{j}, d_{j}^{l}\right)= \begin{cases}\frac{\sigma_{s}\left(d_{j}\right)}{\varepsilon}, & \left|d_{j}^{l}-d_{j}\right|<\frac{\varepsilon}{2} \\
\frac{\sigma_{s}\left(d_{j}\right)}{\varepsilon} \exp \left(-\frac{\left|d_{j}^{l}-d_{j}\right|-\frac{\varepsilon}{2}}{\tau}\right), & \text { otherwise }\end{cases}
\end{gathered}
$$

and $d_{j}$ is the distance between the sensor and the detected obstacle, $d_{j}^{l}$ the distance between the sensor and the voxel's centre, and following the linear model $\sigma(d)=\sigma_{\min }+\zeta . d$. The model states that $\sigma(d)$ is minimum and equal to $\sigma_{\min }$ near to the sensor and increases linearly with distance $d$ with a derivative $\zeta$. This solution relies on sensor calibration to estimate global values for sensor model parameters $\sigma_{\min }$ and $\zeta$.

Using different sensors, the joint distribution decomposition of the relevant variables shows the dependency assumptions according to Bayes' rule as follows:
$P\left(O c Z_{\text {vision }} Z_{\text {grasp }}\right)=P(O c \mid C) P\left(Z_{\text {vision }} \mid O c\right) P\left(Z_{\text {grasp }} \mid O c\right)$

and the posterior is the probability distribution on the occupation's percentage $P\left(O_{C} \mid Z_{\text {vision }} Z_{\text {grasp }}\right)$ for each voxel:

$$
P\left(O c \mid Z_{\text {vis }} Z_{\text {grasp }}\right)=\alpha P\left(Z_{\text {vis }} \mid O c\right) P\left(Z_{\text {grasp }} \mid O c\right) P(O c)
$$

In our previous work [17], entropy $H$ was used to combine two classes of features using Bayesian models to classify reach-to-grasp movements. After analyzing a set of results of different classifiers, weights for each type of classifier was assigned through the entropy. Based on that work, we intend to adopt entropy for confidence level of the sensors models to perform the data fusion.

Through Bayesian techniques we can achieve the sensors fusion and the entropy $H$ can be used as confidence level for that. A confidence variable will be used as weight $\boldsymbol{w} \in\left\{w_{1} \ldots w_{S}\right\}$ for each sensor. The weight $\boldsymbol{w}$ can be expressed as a prior $P(w)$ in the Bayesian rule. For each sensor we can compute the entropy of the posterior probabilities as follows:

$$
H(P(O c \mid Z))=-\sum_{i} P\left(O c_{i} \mid Z\right) \log \left(P\left(O c_{i} \mid Z\right)\right)
$$

where $P(O c \mid Z)$ represents the posterior probability of the occupancy of each cell in the map achieved by a specific sensor. The variable $i$ is the index of each grid cell. Through the entropy $H$ we can achieve the probability distribution of the weights of each sensor. The weights are computed as follows:

$$
w=1-\frac{H c}{H}
$$

where $\boldsymbol{w}$ is the weight result; $H_{C}$ is the current value of entropy that is being transformed in a weight; $H=\sum H_{i}$ where $i$ is the index for each entropy value computed by (10).

Given the confidence of occupancy achieved by each sensor we can fuse the sensors belief using (9) multiplying by the sensors weights reached by the entropy. For each cell of the volumetric map we can compute the sensor fusion belief:

$$
P\left(O c \mid Z_{1}, \ldots, Z_{S}\right)=\sum_{i=1}^{S} P\left(w_{i}\right) P\left(O c \mid Z_{i}\right)
$$

where $i \in S=\{1 \ldots N\}$ representing the amount of sensors.

Using (10) is possible to update a global map with the probability distribution of each cell achieved by different sensors for data fusion. Employing entropy as confidence level we will be sure of the confidence of each sensor, that is, which is more reliable and then it is possible to build the global map from local maps (vision and in-hand exploration) with more certainty of the occupancy of each cell given the measures of the sensors. The only concern that needs to be taken into consideration on using the proposed methodology is the computational cost since using equation (9) for fusion 
will be faster than computing (10)-(12) since it is necessary to compute for each cell of the map.

\section{Frame of Reference for Object-Centric Representation}

To have a better representation of the object, beside of working in an egocentric way (sensor frame of reference) we are adopting an object-centric representation. To find the object frame of reference, the center of mass of the object is computed. The center of mass of the object can be found after the in-hand exploration. We compute the center of mass of the point's distributions (full voxels) that represents the object shape. The 3D moments invariants are a measure of the spatial distribution of the mass of a shape. The center of mass is achieved computing the central moment (summation of the voxels probabilities); the first moments for $\mathrm{x}, y, z$ and then the normalization of the first moments to find the centroid of the object shape.

\section{RESUlts}

\section{A. Single Hand Exploration of Static Objects}

Two objects were used for the single hand exploration (Fig.3). In Fig.4 the raw data of the in-hand exploration is shown and in Fig.5 the computed volumetric map. The darker voxels represent the cells with higher probabilities. This figure shows the representation of the voxels with different thresholds to consider the cells are full (considering all voxels with probabilities $>0$, voxels $>0.6$, voxels $>0.8$ respectively).

Fig.6 shows the 3D point cloud and the correspondent texture extracted from stereo camera; in-hand exploration data with the correspondent texture and the volumetric map with cells with occupancy probability $>0.8$. To extract the texture, images from a stereo camera were taken. We have used SVS library (Small Vision System) from Videre Design [10]. It provides functionalities to compute $3 \mathrm{D}$ points. For the disparity computation, the SVS library uses Laplacian of Gaussian image filter; correlation: sum of absolute differences over a square window; disparity search from 8 to 128 pixels; subpixel interpolation to $1 / 16$ pixel. Through the calibration between the sensors was possible to find the correspondence of one frame of reference to another (Polhemus to stereo camera and vice-versa). The methodology of calibration is presented in our previous work, for more details see [18].

Fig.7 (a) shows the raw data from in-hand exploration of a bottle with the correspondent texture of the camera and (b) shows the object centric representation: correspondent points from stereo camera (texture) and the probabilistic representation.
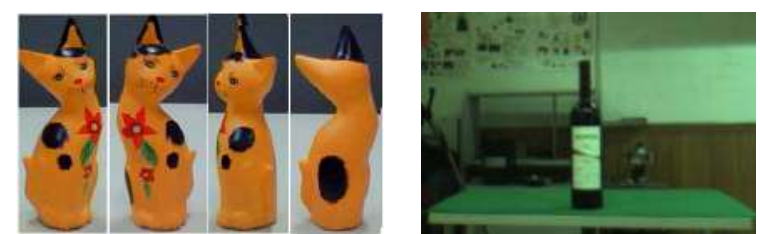

Fig.3. Objects that were used in the experiments. Left image: Different views of the wooden cat; Right image: bottle of wine.
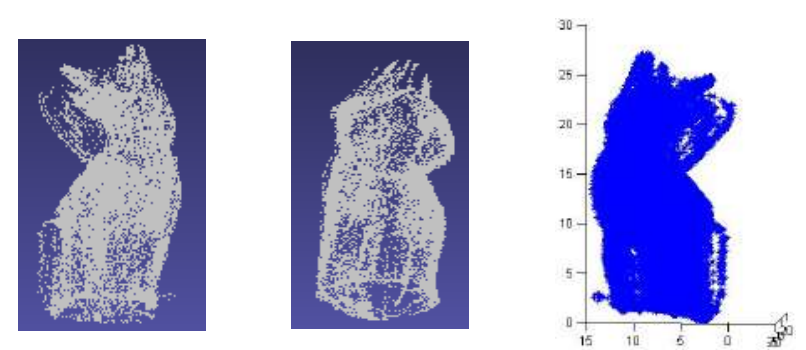

Fig.4. Raw data of the wooden cat acquired from the single-hand exploration

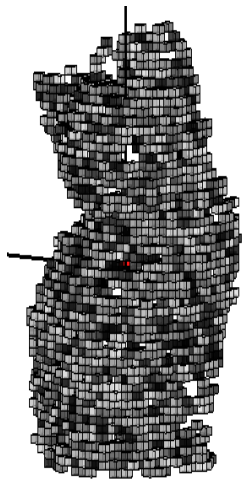

(a)

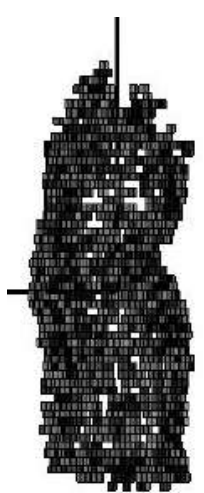

(b)

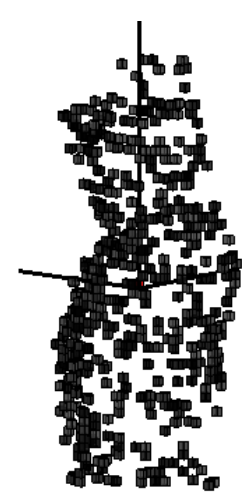

(c)
Fig.5. (a) Computed Probabilistic Map of the wooden cat: Object centric representation

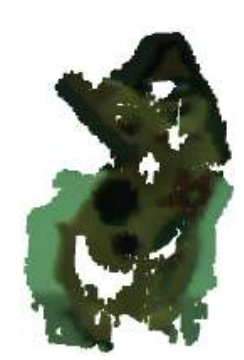

(a)

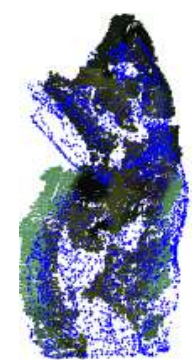

(b)

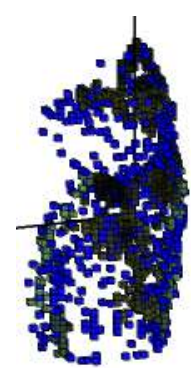

(c)
Fig.6.(a) Extracted texture; (b) Raw data; (c) Prob. Map (prob. > 0.8).

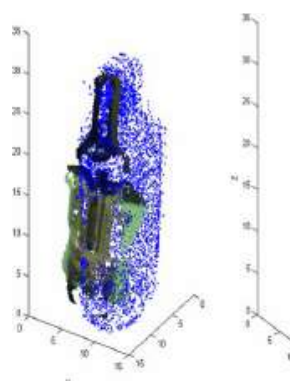

(a)

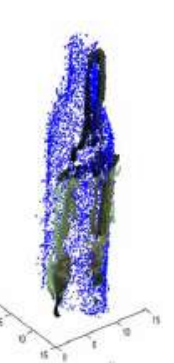

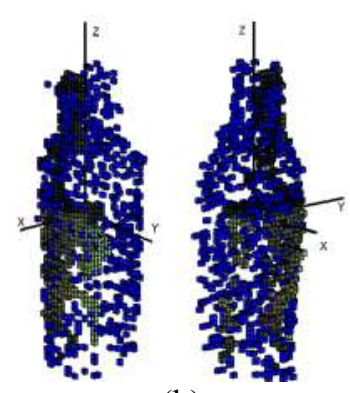

(b)
Fig.7 (a) Views of the registration of in-hand exploration raw data and the

\section{B. In-Hand Exploration of non-Static Objects}

Fig.8 shows the raw data of in-hand exploration of nonstatic object; the registration of the point cloud to a common frame of reference and the computed probabilistic map. Even after the registration of the points to a common frame of reference we still have some points out of the object surface. Probably this happens due to the errors along the angles measure (sensors noise). Using the probabilistic map, it is possible to reduce the noise just considering the cells 
with higher probabilities, e.g. higher than 0.8. Another alternative to solve the orientation problem is to attach two magnetic sensors to the object to reduce the orientation errors.

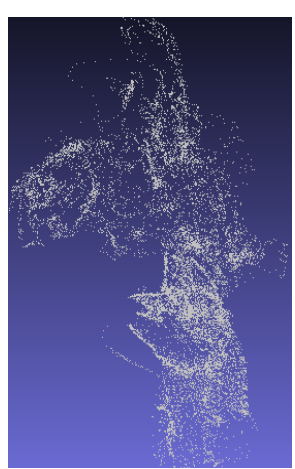

(a)

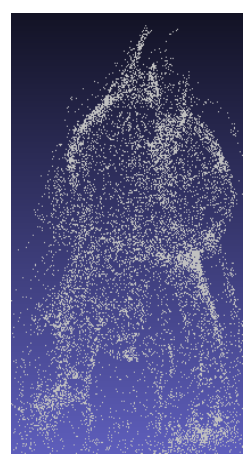

(b)

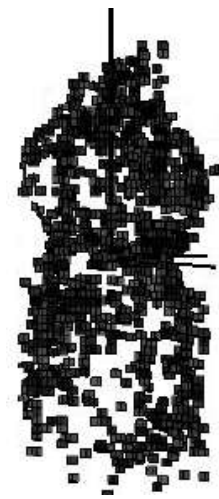

(c)
Fig.8.(a) Raw data of non-static object from the in-hand exploration of wooden cat; (b) the point cloud after registration to a common frame of reference and (c) computed probabilistic map cells with probability $>0.8$.

The results show that valid objects models can be obtained. The volumetric map also provides information concerning the contact points and the most explored region of the object. Even with the limitations of single hand exploration of static objects, it is possible to reduce some noise caused by involuntary movements out of the object surface along the exploration by ignoring cells with low occupancy.

\section{CONCLUSION AND FUTURE WORK}

A probabilistic representation for $3 \mathrm{D}$ objects was presented and shown how given the sensors measurement of in-hand exploration the probabilistic map is computed. The center of mass of the object is found to define the object frame of reference for object-centric representation. Two ways of in-hand exploration are presented: single hand exploration of static objects and in-hand exploration of nonstatic objects. The results show that it is possible to achieve valid models of the object surface. We can deal with the problem of moving object along the in hand-exploration making the registration of the points cloud to the same frame of reference, but we still can find some problems such as noise in the angles measure. We have introduced data fusion models (probabilistic map) where different sensors can be used with the in-hand exploration data to be employed in future applications. As future work we intend to develop and test the stereo vision sensor model presented in section III (C) to acquire 3D point's distribution to be used in a local map. A local map for each sensor will be developed and later the data fusion will be done in a global map. We intend to develop the steps shown in Fig.1, developing methods to extract primitives to find object graspable parts and then perform grasp generation tasks.

\section{REFERENCES}

[1] F.N. Newell, M.O. Ernst, B.S. Tjan, and H. Bülthoff, "Viewpoint Dependence in Visual and Haptic Object Recognition", Psychological Science, vol. 12 (1), 2001, 37-42.

[2] R.L. Klatzk and S. Lederman, "Intelligent Exploration by the Human Hand”. Dextrous Robot manipulation. ed.S.T. Venkataraman and T. Iberall Springer-Verlag, 1990 Cha. 4.

[3] P. K. Allen, K.S., Roberts, "Haptic object recognition using a multifingered dextrous hand". In: IEEE International Conference on Robotics and Automation,1989 342-347.

[4] A. Bierbaum, K. Welke, D. Burger, T. Asfour, R. Dillmann, "Haptic Exploration for 3D Shape Reconstruction using Five-Finger Hands" In IEEE/RAS International Conference on Humanoid Robots. 2007.

[5] A. Bierbaum, I. Gubarev, R. Dillman, "Robust shape recovery for sparse contact location and normal data from haptic exploration". In: IEEE/RSJ International Conference on Intelligent Robots and Systems. IROS'08, 3200-3205.

[6] A. Okamura, M.L. Turner, M.R. Cutkosky, "Haptic Exploration of Objects with Rolling and Sliding", Proceedings of the 1997 IEEE International Conference on Robotics and Automation, April 20-25, Albuquerque, NM, pp. 2485-2490

[7] A. Okamura and M. Cutkosky, "Haptic exploration of fine surface features", Proc. of IEEE Int. Conf. on Robotics and Automation, ICRA'99, pp. 2930.2936, 1999.

[8] I. Biederman, "Recognition-by-Components: A Theory of Human Image Understanding”, Psychological Review, 1987, vol.94, 115-147.

[9] Polhemus Liberty Electromagnetic Motion Tracking System: http://www.polhemus.com

[10] Videre Design: http://www.videredesign.com/vision/sth_mdes3.htm

[11] H. Moravec, "Sensor fusion in certainty grids for mobile robots". AI Mag. 9, 1988 61-74.

[12] A. Elfes, "Using occupancy grids for mobile robot perception and navigation". Computer 22, 1989, 46-57.

[13] S. Thrun, "Robotic mapping: a survey". In: Exploring artificial intelligence in the new millennium. Morgan Kaufmann Publishers Inc., San Francisco, CA,USA, 2003, 1-35.

[14] J. Ferreira, C. Pinho, J. Dias, "Active exploration using bayesian models for multimodal perception". In: ICIAR '08: Proceedings of the 5th International conference on Image Analysis and Recognition, Berlin, Heidelberg, Springer-Verlag, 2008, 369-378.

[15] D. R. Faria, R. Martins, J. Dias , "Grasp Exploration for 3D Object Shape Representation using Probabilistic Map" In Proceedings Doctoral Conference on Computing, Electrical and Industrial Systems, Costa da Caparica, Portugal, February, 2010.

[16] R. Rocha, J. Dias and A. Carvalho, "Exploring information theory for vision-based volumetric mapping", IEEE/RSJ International Conference on Intelligent Robots and Systems, 2005, pp. 1023- 1028.

[17] D.R. Faria and J. Dias, "3D Hand Trajectory Segmentation by Curvatures and Hand Orientation for Classification through a Probabilistic Approach". In Proceedings of The 2009 IEEE/RSJ International Conference on Intelligent Robots and Systems, IROS'09, St. Louis, MO, USA, October 2009.

[18] D.R. Faria, H.Aliakbarpour and J. Dias, "Grasping Movements Recognition in 3D Space using a Bayesian Approach" in Proceedings of 14th International Conference on Advanced Robotics - Munich, Germany, 2009. 